\title{
CT Image Management and Communication Services
}

\author{
Kanda Runapongsa Saikaew, Nantachai \\ Jirandorn, Nuntapon Juntaranon, Supree \\ Ariyadech \\ 1Department of Computer Engineering \\ Khon Kaen University, Thailand \\ krunapon@,kku.ac.th
}

\author{
Vera Sa-Ing, Wasan Pattara-Atikom, Saowapak S. \\ Thongvigitmanee \\ National Electronics and Computer Technology Center \\ Pathumthani, Thailand \\ saowapak.thongvigitmanee@,nectec.or.th
}

\begin{abstract}
Nowadays computed tomography (CT) becomes very popular and useful in both medical and dental areas. Due to the standard DICOM (Digital Imaging and Communications in Medicine) file format of CT images containing special headers of image, patient and device information, dentists or specialists require to use DICOM viewer software to display those images. After acquiring a CT scan, a traditional way to transfer the CT images from the CT workstation to another place is by having someone carry a CD containing DICOM images with or without viewer software. To accommodate or speed up this process, especially when an interpreting radiologist and a referring dentist are in different places, we have developed a CT image management and communication service process which provides online dental image management as well as viewer software available for the invocation by web and mobile applications. Our system can support image stream, online $C T$ scan request and image download services. The web and mobile applications allow dentists or specialists to view and analyze images at anywhere anytime. This complete system would gain high benefits to not only a dental CT service center but also other medical imaging service centers as well.
\end{abstract}

Keywords-Computed Tomograph; DICOM; Viewer software; Medical diagnostics

\section{INTRODUCTION}

In general, after acquiring a CT-scan, the digital image files in Digital Imaging and Communications in Medicine (DICOM) format are stored on a database. The DICOM images are usually transferred to dentists or specialists through a CD. This process is inconvenient. The Internet can play an important role in accessing data remotely and simultaneously. Compared with a standalone version viewer software, the web application viewer can provide similar basic features but with much fewer costs and wider access.

Several new web application technologies exist to ease the use and contribute to the popularity of web applications. These technologies include HTML5, JavaScript, and CSS3. HTML5, the newest version of HTML, is an interesting markup language. A new JavaScript graphics library called canvas brings a lot of animation effects that previously were only available via Adobe's Flash, thus plugin is unnecessary. HTML5 and JavaScript help users to interact with the web application naturally similar to what they can do with the desktop application. Cascading style sheet version 3 can adjust the user interface of web application in a great look and feel.

Nowadays more people access web application and other Internet resources via mobile devices. In 2011, more than $50 \%$ of all local searches were taken from mobile phones and it is predicted that mobile Internet usage will overtake desktop usage in 2014 [5]. Apple iPad is one of the bestselling devices because of a high number of apps available in the Apple App Store. It is reported that Apple currently owns about $75 \%$ of the tablet market and is likely to dominate this market until 2015 [6].

WADO (Web Access to DICOM Object) service specifies a web-based service for accessing and presenting "DICOM persistent" objects (images and reports). This service connects with PACS (Picture Archiving and Communication System). Web-based PACS has "thin clients" which means clients need only a browser to access the server and no special hardware is required. Only a browser is needed to view images. All functions (window width and level, zoom, etc.) are typically provided as controls within the server application. Images are delivered to the client PCs only on demand, and the browser does not locally store the images.

In this paper, we propose the image management services that provide Application Programming Interface (API) for various types of client applications. We have implemented both web and iPad apps as client applications but the provided API can also be invoked by other types of client applications. Unlike conventional PACs, the proposed services and apps have two main advantages which are 1) the developed API can be invoked by any client applications not only by a browser and 2) the developed iPad application can access and manipulate images while offline.

With the proposed API, the applications can display images and have basis features for CT image viewer. Dentists or specialists can access images instantly when an administrator finishes uploading. Dentists or specialists do not need to be at the CT center. They can access images at 
anywhere anytime. Patients who have an emergency case will be treated urgently.

\section{RELATED WORK}

In 2011 Pablo Sau released the first Debian packages of DCM4CHEE software (Open Source Clinical Image and Object Management) which can be downloaded at http://sourceforge.net/projects/cdmedicpacsweb/files/ [8] After installing such provided files at a Linux machine, the user will have a web with full featured free PACS based on dcm4chee and mysql, with remote web accession available. Several iPad radiology viewers are now available, such as OsiriX HD, Aycan xr.ay, Merge \& ResolutionMD, but MIM is the first one that is a cloud-based DICOM service [9]. Fernandez-Bayo et. al. proposed to distribute medical images via a DICOM Web server and a DICOM Java Viewer [10]. Unlike these existing works, we also provide APIs that others can use to develop other customized applications. In this paper, we also have implemented two applications (Web app and iPad app) by invoking the provided APIs.

\section{Methodology}

We create a new web API that allows a variety of software clients, such as web and iPad applications. The API provides a comprehensive and complete for medical image services. A doctor can fill in a request from the web application and wait for a patient to take a CT scan. Then images will be uploaded by an administrator. After being uploaded, images will be processed and prepared for being downloaded or accessed by a viewer. A medical staff can use the viewer application via web or iPad apps to view images wherever the Internet is available as shown in Figure 1. Furthermore, some functions on iPad app can be accessible when the user is offline. These functions include displaying images, zoom/pan and distance measurement.

In this section we will discuss the image service which is available as REST API that can be invoked by the client programs such as web and iPad apps.

\section{A. REST API}

The web API at the server is responsible for storing images from a CT scan, displaying and processing images, and supporting for scan requests. The API is then invoked by the web application which displays images on a web browser and by the iPad application as shown in Figure 2. These two clients connect to the server via HTTPS for a security reason and using the HTTP POST method to send and upload the new data at the server.

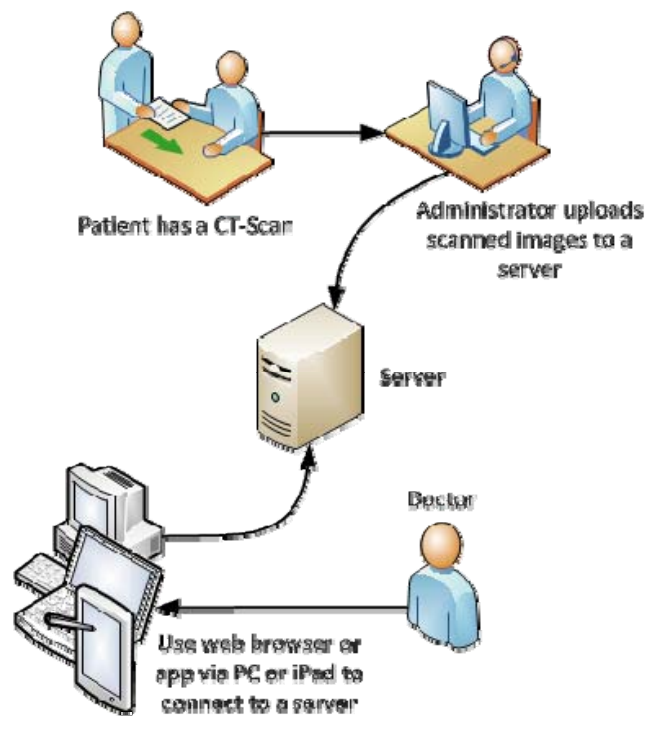

Figure 1. The system usage.

REST API is written using Java language with Oracle Glassfish Server as a web application server. The REST API uses HTTPS protocol with the POST method. An external request from doctors will be detected and have access granted by a server. The parameters from viewer application will be sent in the URL to the server. Images in JPEG format will be sent from the server to the viewer application.

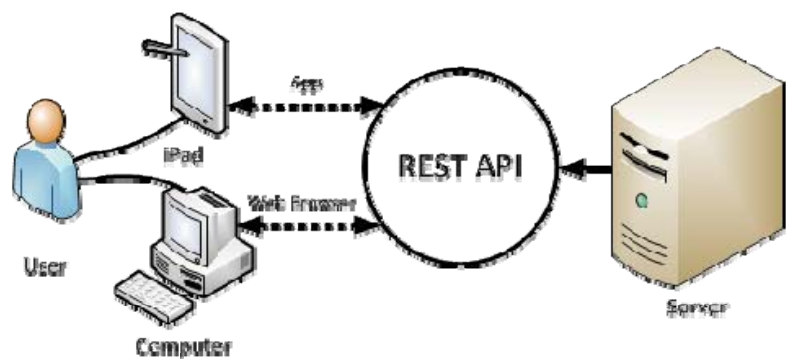

Figure 2. A server provides the REST API to clients.

All functions at the server are listed as follows.

- Administrator tool

- Add User: Add a user to a system and identify an access level

- Edit User: Edit information of a user

- Remove User: Remove information of a user from a system

- Configure File Storage: Location to store a file

- Authentication

- Authenticate Session: Verify a session that is used in system

- Destroy Session: Delete a session in a system 
- Session Filter: Filter each session that accesses to a system

- Upload: Upload an image file from an upload page and add information of an image file into a database

- DICOM download: Download a DICOM file that is compressed

- DICOM tag: Read information in a DICOM tag

- JPEG stream: Convert a DICOM file to JPEG for display on a device

- Reslice: Process a multiplanar view from a DICOM axial view

- Search: Search a patient study list

- Scan request: Scan request form for having a CT scan

Details of functions available in REST API are as follows.

- Login API: User request to login

URL: $\quad$ ip:port/CTmanagement/Login

Parameter: username,password

Return: Login successfully

Login fail

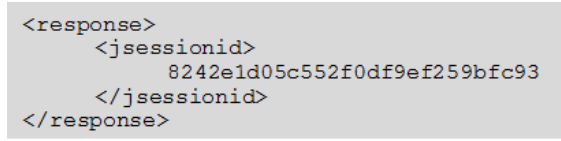

Figure 3. Example of a successful login.

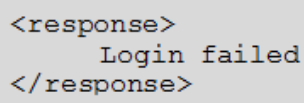

Figure 4. Example of a fail login.

- Logout API: User request to logout

$\begin{array}{ll}\text { URL: } & \text { ip:port/CTmanagement/Logout } \\ \text { Parameter: } & \text { JSESSIONID } \\ \text { Return: } & \text { Invalidated session }\end{array}$

<response>
Invalidate session : 8242 e1d05c552f0df9ef259bfc 93
</response>

Figure 5. Example of an invalidated session.

- Search Study List API: Request an information of patient

URL:

Parameter:

ip:port/CTmanagement/Search

Return:

Patient's study list

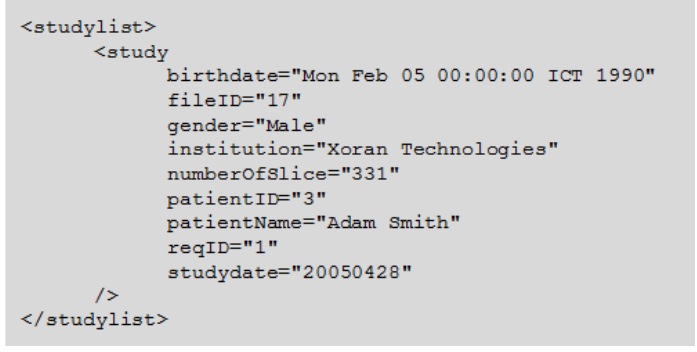

Figure 6. Example of a patient's study list.

- Get DICOM Tag API: Request information of DICOM tag

URL: ip:port/CTmanagement/DICOMTag

Parameter: JSESSIONID, filesid

Return: DICOM tag

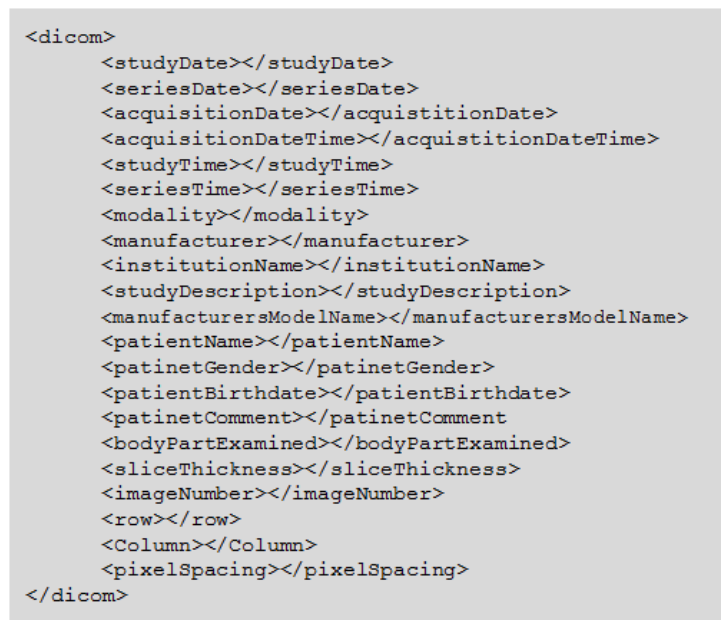

Figure 7. Example of a DICOM tag.

- Get User Information API: Request information of user and check an session expired

URL: ip:port/CTmanagement/UserInfo

Parameter: JSESSIONID

Return: User information

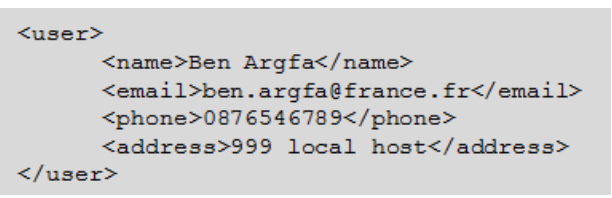

Figure 8. Example of a user information.

- Get Image API: Request an image from a server URL: ip:port/CTmanagement/JpegStream Parameter: JSESSIONID, filesid, slicenumber, ww (window width), wl (window level)

Return: An image related to the values from parameters 


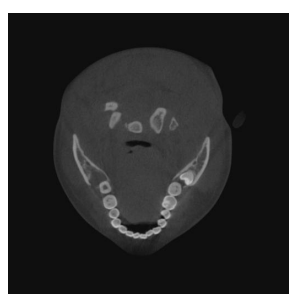

Figure 9. Example of an image from using a get image API.

- Get multiplanar image API: Request an image which is sagittal or coronal view from a server URL: ip:port/CTmanagement/Reslice

Parameter: JSESSIONID, filesid, ww (window width), wl (window level),x (image's position in $\mathrm{x}$ axis), $\mathrm{y}$ (image's position in y axis), cor (result's planar (xz, yz))

Return: An image in other views related to the values from parameters

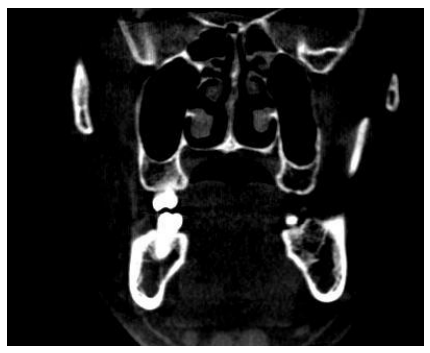

Figure 10. Example of an image from using get multiplanar image API.

- Download Files API: User request to download a DICOM file that is compressed in a zip file

URL:

ip:port/CTmanagement/Download/filena

me.zip

Parameter: JSESSIONID, reqID (Received from Get Study API), type (Type of image: dem or png)

Return: A compressed images package in Zip format

$$
\text { Download.zip }
$$

Figure 11. Example of a Zip file using download files API.

- Scan Request API: User request to scan URL: ip:port/CTmanagement/ScanRequest Parameter: JSESSIONID, pat_fn (Patient's name in English) pat_ln (Patient's surname in English), pat_fn_th (Patient's name in Thai), pat_ln_th (Patient's name in thai), pat_birthdate (Patient's birthdate in format 'yyyymmdd'), pat_gender (Patient's gender), pat_phone (Patient's telephone number), date (Date of form request was submitted in format 'yyyymmdd'), purpose (String), section (String), attached_instrument (String), interpretation (String),

clinical_information (String), delivery_method (String), specific_tooth (String)

Return: Status inserting a new data in a database

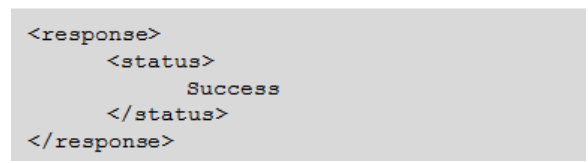

Figure 12. Example of a status if a data is valid.

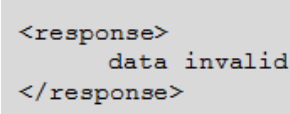

Figure 13. Example of a status if a data is invalid

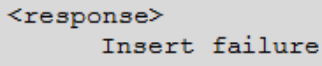

Figure 14. Example of a status if server cannot insert a data in database.

\section{B. Web application}

The web application is on the HTML platform using HTML5 features which are canvas elements to construct a view. Due to a power of JavaScript, web application can be created dynamically and flexibly. A viewer web-based application provides standard functions as in the viewer software such as DICOM tag reader, zoom/pan, window/level adjustment, and measurement tools. Parameters will be sent to a server via a URL. Zooming and moving images as well as measurement tools are processed on the web application side. Calculating distances and angles with basic mathematics is based on a scale of the image. Window and level values (aka brightness and contrast) are sent via API to request images with the specified parameters. The images will be temporarily stored in a browser cache until the session ends.

\section{C. iPad applicaiton}

The iPad application is written in Objective-C language. The overall feature is similar to the web application. But the iPad application can be accessible when the user is offline. Since images are downloaded and stored on the iPad, they can be viewed later.

Techniques developed in each function are as follows. The image download function uses the NSURLConnection class to connect to the server and NSXMLParser class to parse the XML file that contains the DICOM tag. The display image function uses the UIImageView class to display a patient's images. The zoom/pan function uses UIScrollView class to zoom and pan an image. The distance measurement function uses the Pythagoras' theorem to 
calculate the distance from one point to another point based on an image scale.

\section{EXPERIMENTAL RESULT}

The REST API mentioned above is used in many functions of both web and iPad applications. Some APIs are not used in the viewer function but used in the system authentication and the scan request function. Some viewer functions, such as measurement tools are processed in the client side. The server side manages user information and identifies an access level of a user. The viewer functions are listed as follows.

\section{A. Web application's function}

- $\quad$ Read DICOM Tag: Read a DICOM tag from the $\mathrm{XML}$ file that is created by server. The result is information of an image including patient's name, patient's surname, patient's birthdate, patient's gender, patient's telephone number and date of scan request was submitted (Figure 15).

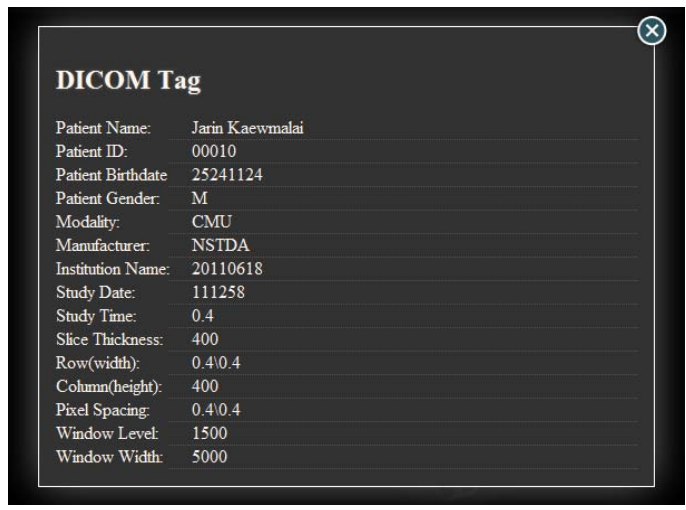

Figure 15. Display DICOM tag function on the web application.

- Zoom/Pan image: Zooming in and out and moving an image by using a mouse. The maximum value can be adjusted (Figure 16).

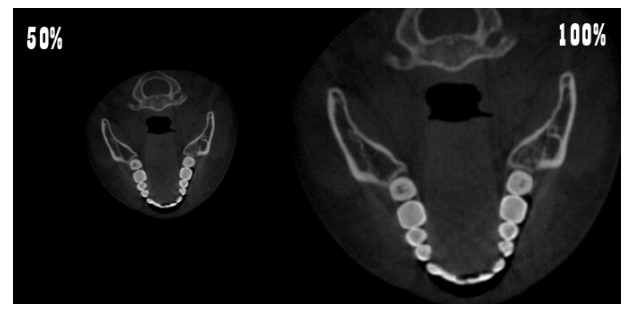

Figure 16. Zoom function on the web application.

- Measurement tools

o Ruler: Always display, stretch and shrink along the zoom/pan function

o Distance measurement: Measure a distance between one point and another point by mouse (Figure 17)
- Angle measurement: Measure an angle in a degree unit (Figure 17)

- Delete tools: Delete all displayed measurement tools

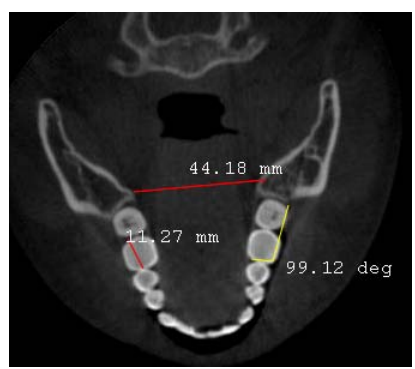

Figure 17. Measurement function on the web application.

- Window width/Window level tools: Adjust Brightness and contrast of an image

○ Window width: Adjust contrast of an image (Figure 18)

○ Window level: Adjust brightness of an image (Figure 18)

- Reset window width/window level: Reset the value of the window width and window level to the value of an original image

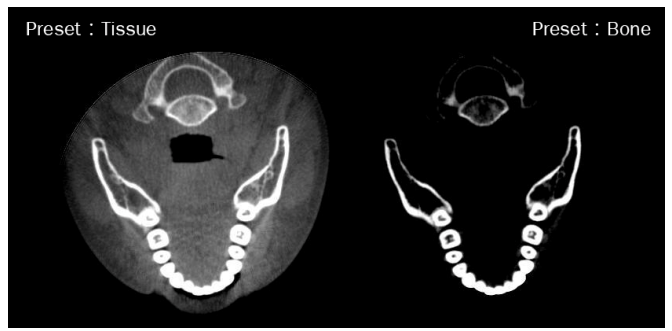

Figure 18. Window/level function on the web application.

- Change view mode: Change a view

○ Single view: Display an image in an axial view

- Sequence view: Display an image in a sequence view

○ Multiple view: Display an image in multiplanar views (axial, coronal and sagittal)

\section{B. iPad application's function}

- Data management: User can remove data

- DICOM Tag: Display the information of the DICOM Tag (Figure 19) 


\begin{tabular}{l} 
Patient name: Jarin Kaewmalai \\
Patient ID: 00010 \\
Gender: M \\
Birthdate: 25241124 \\
\hline$-a-a$ Modality: CMU \\
Manufacturer: NSTDA \\
Institution name: 20110618 \\
Studydate: 111258 \\
Studytime: 0.4 \\
\hline Slice thickness: 400 \\
Pixel spacing: 0.410 .4 \\
Row: 0.410 .4 \\
Column: 400
\end{tabular}

Figure 19. Display the DICOM tag on the iPad application.

- Zoom/Pan: Pinch to zoom in and zoom out and pan to move an image

- Brightness and Contrast: Adjust brightness and contrast of an image

- Distance measurement: Measure a distance from one point to another point (Figure 20)

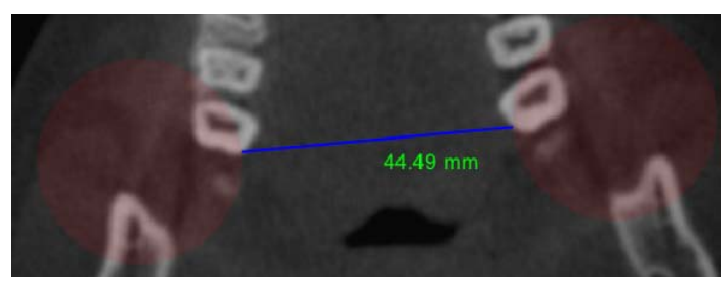

Figure 20. Distance measurement function on the iPad application.

The web application can be accessed in a single view (Figure 21), a sequence view (Figure 22. Sequence view. and multiplanar views including axial, sagittal, and coronal views (

Figure 23) by using change view function. The iPad application can display the DICOM tag and measure a distance of an image (Figure 24-25).

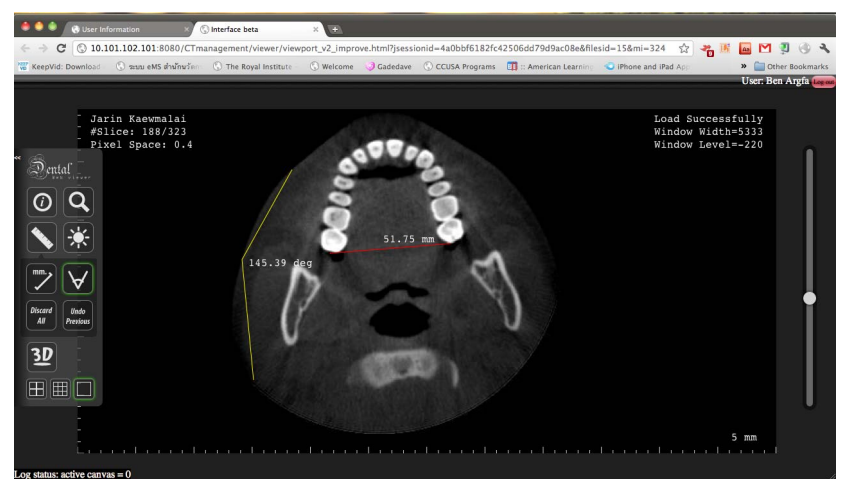

Figure 21. Single view (Axial view).

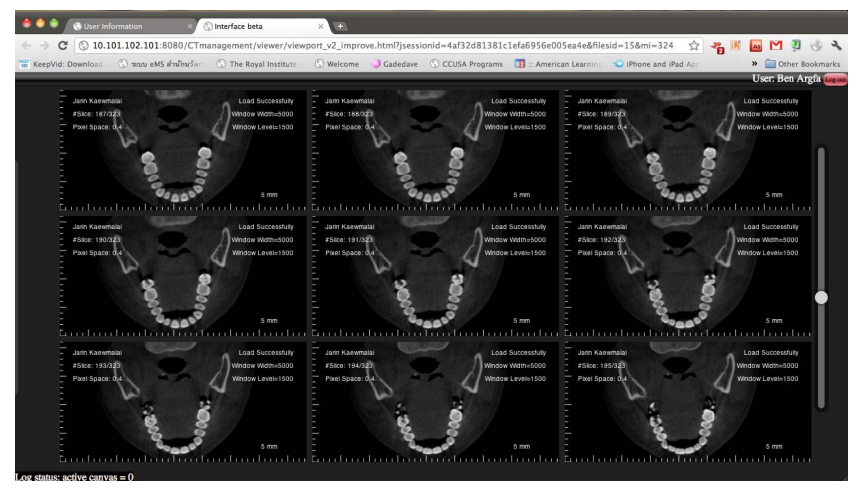

Figure 22. Sequence view.

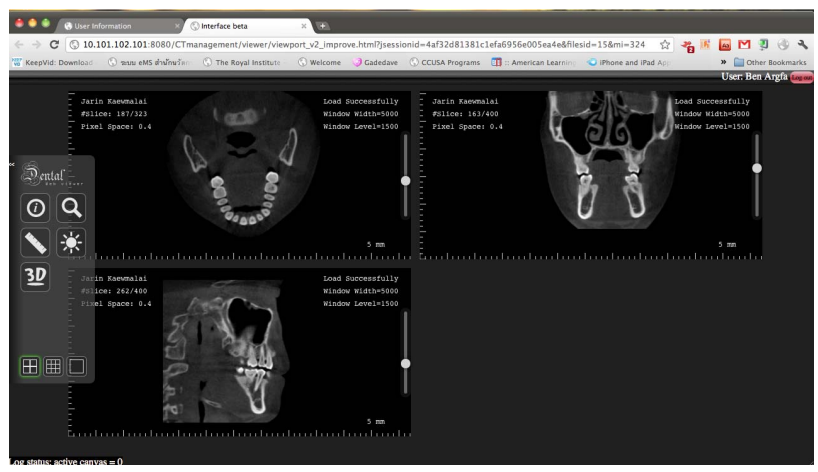

Figure 23. Multiplanar views.

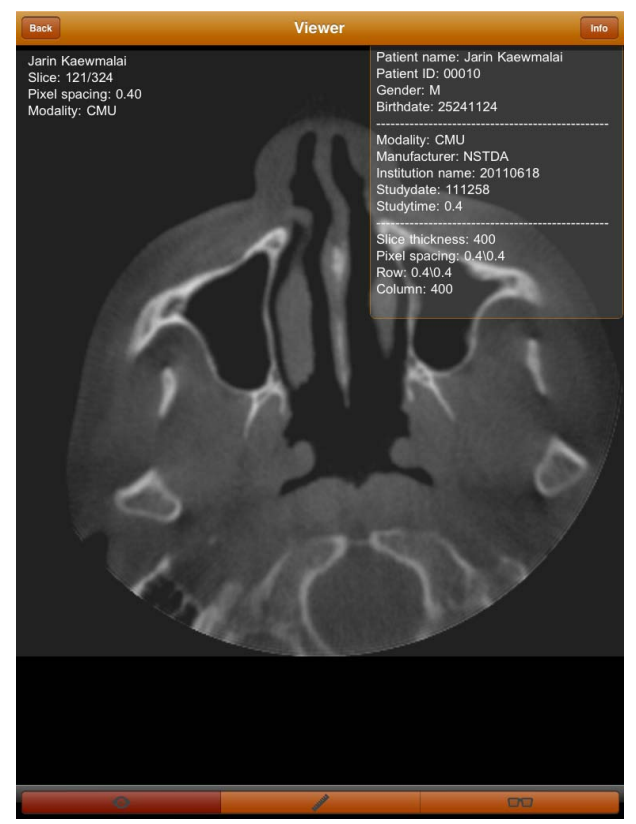

Figure 24. Display an image and display the DICOM tag on the iPad application. 


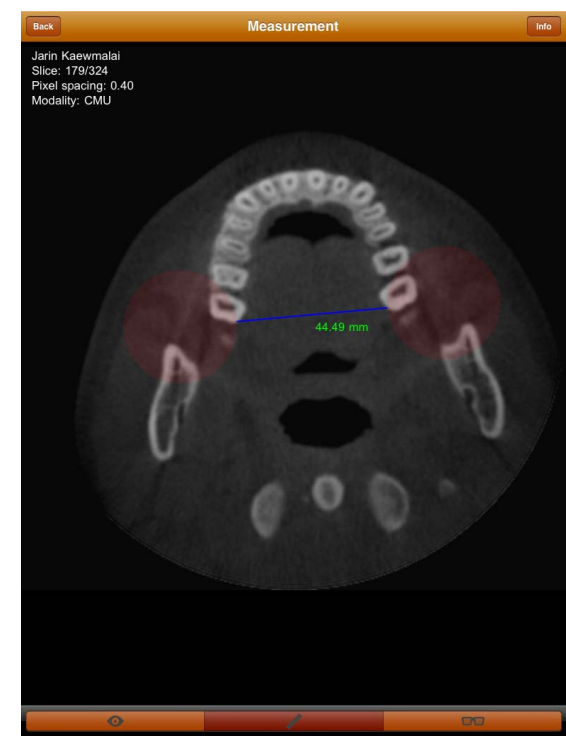

Figure 25. Distance measurement function on the iPad application.

\section{CONCLUSION}

The CT image management and communication services consists of 3 parts: REST API, web application and iPad application. CT images are processed at the server side which manages API authentication and provides REST API. The web application can display images that are the output of the REST API functions. These functions allow the users adjust contrast and brightness, and display images in a single view, sequential views, and multiplanar views. In addition, it also allows the users to perform distance measurement, angle measurement and DICOM tag display. The iPad application has similar functions as available on the web application. However, CT viewer application on iPad can be used even in areas without Internet. Offline functions on the iPad application provide the same functionality as when online. Our service and client applications allow dentists/physicians to diagnose and plan the treatment more easily and quickly. Further development of the service is to support 3D display.

\section{ACKNOWLEDGMENT}

We would like to thank the DentiScan team at National Electronics and Computer Technology Center (NECTEC) for their guidance and technical support. This project is financially supported by the Young Scientist and Technologist Program (YSTP: SP54-NT04 and SP54NT05) from National Science and Technology Development Agency as well as from the National Software Contest (NSC) which our project won the third prize in the category of programs for the development in science and technology.

\section{REFERENCES}

[1] Fulton S, Fulton J. HTML5 Canvas. California: O’Reilly Media, Inc.; 2011.

[2] Paireepairit I. The iPhoneTM Developer's Cookbook. Bangkok: Asiaplace; 2008. (in Thai).

[3] Pulam R. iPhone Apps Handbook. Bangkok: Provision; 2010. (in Thai).

[4] Wahli U, Fielding M, Mackown G, Shaddon D, Hekkenberg G. Servlet and JSP Programming with IBM WebSphere Studio and VirtualAge for Java. California: IBM Corporation; 2000.

[5] Digitalbuzzblog. Infographic: Mobile Statics, Stats \& Facts 2011. [online]. Available: http://www.digitalbuzzblog.com/2011-mobilestatistics-stats-facts-marketing-infographic/

[6] Lauren Indvik. iPad To Dominate Tablet Market Until 2015. [online]. Available: http://mashable.com/2011/07/27/tablet-study-ipad-android/

[7] George V. Koutelakis and Dimitrios K Lymperopoulos, "PACS through Web Compatible with DICOM Standard and WADO Service: Advantages and Implementation" $28^{\text {th }}$ IEEE EMBS Annual International Conference, New York City, USA, Processings, pp. 98104, Aug 30-Sept 3,2006.

[8] Pablo Sau. How to Deploy a Web PACS in Minutes. [online]. Available:

http://cdmedicpacsweb.sourceforge.net/CDMEDIC_PACS_WEB.htm 1

[9] Felasfa Wodajo. Mobile MIM liberates doctors to view CT \& MRI scans on iPads and is FDA approved. [online]. Available: http://orthoonc.com/2011/05/15/mobile-mim-liberates-doctors-toview-ct-mri-scans-wherever-they-are-and-is-fda-approved-appreview/

[10] Fernàndez-Bayó $J$, Barbero $O$, Rubies $C$, Sentís $M$, Donoso L . Distributing medical images with internet technologies: a DICOM web server and a DICOM java viewer. RadioGraphics $2000 ; 20$ ( 2 ): $581-590$ 